\title{
The optimal locations for shock acceleration in MHD jets
}

\author{
Peter Polko $^{1}$, David L. Meier ${ }^{2}$ and Sera Markoff ${ }^{1}$ \\ ${ }^{1}$ Astronomical Department "Anton Pannekoek", University of Amsterdam, \\ Postbus 94249, NL-1090GE, Amsterdam, the Netherlands \\ email: P.Polko@uva.nl and S.B.Markoff@uva.nl \\ ${ }^{2}$ Jet Propulsion Laboratory, California Institute of Technology, Pasadena, CA 91109, USA \\ email: David.L.Meier@jpl.nasa.gov
}

\begin{abstract}
Jets can contribute to the spectra of X-ray binaries (XRBs) and active galactic nuclei (AGN) from the radio through the $\gamma$-ray bands; thus understanding their physics is key for interpreting the data. Recent VLBI observations suggest that jets begin to accelerate particles into power-law distributions at a point offset from the black hole by $\sim 10^{4} r_{\mathrm{g}}$, possibly via a collimation shock. Spectral fitting of simultaneous, broadband data from both XRBs and AGN in jet-dominated states corroborates this picture. From a magnetohydrodynamical (MHD) point of view, it is natural to associate the onset of particle acceleration with the final MHD critical point in the flow, the modified fast point (MFP), where causal contact with the upstream flow is broken. In this way a standing disruption like a shock can form, and this location might vary with the physical parameters of the jet. In order to study this issue, we have used the self-similar formalism of Vlahakis \& Königl (2003, hereafter VK03) to simplify the MHD equations and to derive solutions that cross the critical points. We have found a new parameter space of solutions that cross the MFP at a finite height above the disc and are relativistic, spanning a range of Lorentz factors $\Gamma \leqslant 10$ (Polko et al. 2010). We present these results, as well as preliminary work connecting the relativistic formalism to the non-relativistic conditions with gravity near the base of the jets.
\end{abstract}

Keywords. acceleration of particles, ISM: jets and outflows, MHD, methods: analytical

\section{Introduction}

The assumptions in VK03 to make the MHD equations tractable are self-similarity and axisymmetry, which are also seen in numerical simulations (see figure 2 and 11 from McKinney, 2006), idealised MHD and time-independence. Due to self-similarity, VK03 had to ignore the effect of gravity. Because we want to fit our solutions to a corona model where gravity certainly plays a role, we compared the relativistic VK03 with the nonrelativistic Vlahakis et al. (2000) including gravity and found a relativistic expression for the gravity term.

\section{Results}

We found a solution that smoothly crosses all three critical points (Figure 1). Initially the jet is accelerated thermally (with a corresponding decrease in specific relativistic enthalpy), at later times magnetic acceleration takes over (as shown by a decrease in the Poynting-to-mass ratio). All solutions so far are in the return-current regime $(F=$ $0.75)$ as we have not changed this parameter yet. The solutions span a wide range of properties such as initial temperature, final velocity and magnetisation of the jet allowing a multitude of systems to be described, including but not limited to XRBs and AGN. 
Figure 1. The top panel shows an example solution where the value of the numerator and denominator of the "wind equation" cross zero at the MFP and the modified slow point (MSP) (the Alfvén point, indicated by the vertical line, has been divided out for clarity). The variable $\theta$ is the angle in radians between the radial vector crossing the field line and the axis of symmetry. The y-axis is logarithmic at large values and linear near zero. The accretion disc is located at $\theta=\pi / 2$. The ratio $\left(\mathrm{d} M^{2} / \mathrm{d} \theta\right)$ stays finite, showing it is indeed a smooth crossing. The lower panel shows the Poyntingto-mass flux ratio $(S)$, the specific relativistic enthalpy $(\xi)$ and the Lorentz factor $(\Gamma)$ for this same solution. It can be seen very clearly that the initial acceleration is thermal, leaving the jet cold $(\xi \approx 1)$, and that later acceleration is magnetic. The lines curve back at the right side because the field lines overcollimate, leading to a possible collimation shock. The MFP occurs after overcollimation has begun. The cylindrical radius is scaled to the Alfvén point.
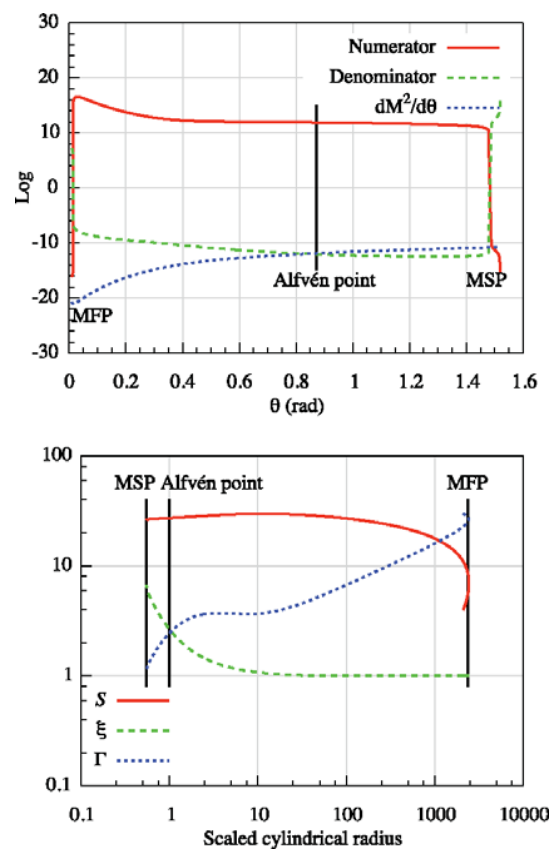

\section{Conclusions \& Future work}

We have shown for the first time that it is possible to obtain solutions to the ideal MHD equations assuming an axisymmetric, self-similar jet that smoothly cross all three critical points. Every solution found overcollimates shortly before the MFP, allowing a shock to develop, that, because it occurs beyond the MFP, cannot disrupt the flow upstream causing the shock, allowing it to be a stable feature in the jet. With the identification of the MFP as the start of the acceleration region, which fits to observations have determined to also be a steady location (e.g., Markoff et al. 2005; Migliari et al. 2007; Markoff et al. 2008; Maitra et al. 2009) but is currently a free parameter, it is therefore possible to independently determine where particles first get accelerated into a power-law distribution. With the wide range in solutions it should be possible to fit the boundary conditions imposed by observations for both XRBs and AGN. After connecting our solutions to a corona model and determining the extent of the possible solutions, we can replace the hydrodynamical acceleration in the spectral fitting routine of Markoff et al. 2005 with the MHD formalism presented here, allowing us to fit the spectra of XRBs more self-consistently and to obtain better estimates for the parameters describing the XRB system.

\section{References}

Maitra, D., Markoff, S., Brocksopp, C., Noble, M., Nowak, M., \& Wilms, J. 2009, MNRAS, 398, 1638

Markoff, S., Nowak, M. A., \& Wilms, J. 2005, ApJ, 635, 1203

Markoff, S., Nowak, M., Young, A., et al. 2008, ApJ, 681, 905

McKinney, J. C. 2006, MNRAS, 368, 1561

Migliari, S., et al. 2007, ApJ, 670, 610

Polko, P., Meier, D. L., \& Markoff, S. 2010, ApJ, in press

Vlahakis, N. \& Königl, A. 2003, ApJ, 596, 1080

Vlahakis, N., Tsinganos, K., Sauty, C., \& Trussoni, E. 2000, MNRAS, 318, 417 